\title{
Una propuesta de innovación docente para la asignatura de Chino V en el Grado de Estudios de Asia Oriental
}

\section{A proposal of teaching innovation for the subject of Chinese $V$ in the Degree of East Asian Studies}

YINGYING XU

Universidad de Sevilla

Departamento de Filologías Integradas

xying@us.es

ORCID: https://orcid.org/0000-0002-1739-0055

DOI: http://dx.doi.org/10.12795/9788447231003.073

Pp.: 1553-1574 


\section{Breve descripción del contexto}

El presente ciclo de mejora ha sido aplicado en la asignatura de Chino V del Grado de Estudios de Asia Oriental. Se trata de una asignatura obligatoria de tercer curso para los alumnos de la mención china. Los alumnos que cursan esta asignatura normalmente ya tenían superadas las asignaturas de Chino I a Chino IV, es decir, que tienen una base de conocimiento del idioma chino equivalente aproximadamente al nivel A2. Sin embargo, debido a la suspensión de las actividades universitarias durante el segundo cuatrimestre del curso pasado 2019-2020 causadas por el Covid-19, los alumnos recibieron pocas clases presenciales y no estaban familiarizados ni acostumbrados a la docencia virtual, por lo cual, estos alumnos presentan un nivel del chino muy heterogéneo, factor que dificulta la elaboración y aplicación del CIMA.

Este grupo cuenta con 28 alumnos matriculados divididos en tres subgrupos, cada semana viene un subgrupo a las clases presenciales y el resto reciben clases de modo virtual. Esta modalidad de docencia hibrida, presenta varias deficiencias para la aplicación del CIMA, como la disminución de la participación en clase, el establecimiento de buenas relaciones y confianza entre los alumnos y el docente y la de no poder ver a los alumnos dificulta la observación de la correcta vocalización, Y en las últimas dos sesiones del CIMA, hemos pasado a la modalidad virtual total, por lo cual, nos encontramos antes una situación muy extraña y poco habitual para la aplicación del ciclo de mejora.

Ciclos de Mejora en el Aula (2020). Experiencias de Innovación Docente de la US Esta obra se distribuye con la licencia Creative Commons 


\section{Diseño previo del CIMA}

\section{Mapa de contenidos}

En las asignaturas de chino del Grado de Estudios de Asia Oriental usamos el libro de texto "El chino de hoy" como el método principal. Sin embargo, este libro no agrupa los temas por sus similitudes, cada lección trata de un tema distinto, es decir que entre una lección y otra no hay una continuidad de secuencias. Hay temas que aparecen varias veces como los de viaje y de comida, y otros temas que sólo aparecen una vez para introducir la gramática o las expresiones idiomáticas de un contexto determinado. Por lo cual, los contenidos están muy dispersos y desordenados. Los alumnos aprenden los temarios y las estructuras gramaticales siguiendo al libro, pero pueden que tengan dificultades para enlazarlos y aplicarlos en las situaciones reales. Por esta razón, he tenido que seleccionar y organizar los contenidos para que tengan continuidad y utilidad práctica en situaciones reales y cotidianas.

Para diseñar el CIMA he escogido tres temas iniciales que se mantengan cierta relación entre si para que el contenido de estas clases prácticas tenga una continuidad. El contenido de esta unidad didáctica consiste en los aspectos lingüisticos y socioculturales relacionados con un viaje de negocios a China, incluyendo la reserva de un hotel, noción básica de aspectos culturales en el negocio y conversaciones sobre viajes realizados y vida cotidiana de un trabajador chino (figura 1).

Ciclos de Mejora en el Aula (2020). Experiencias de Innovación Docente de la US Esta obra se distribuye con la licencia Creative Commons 


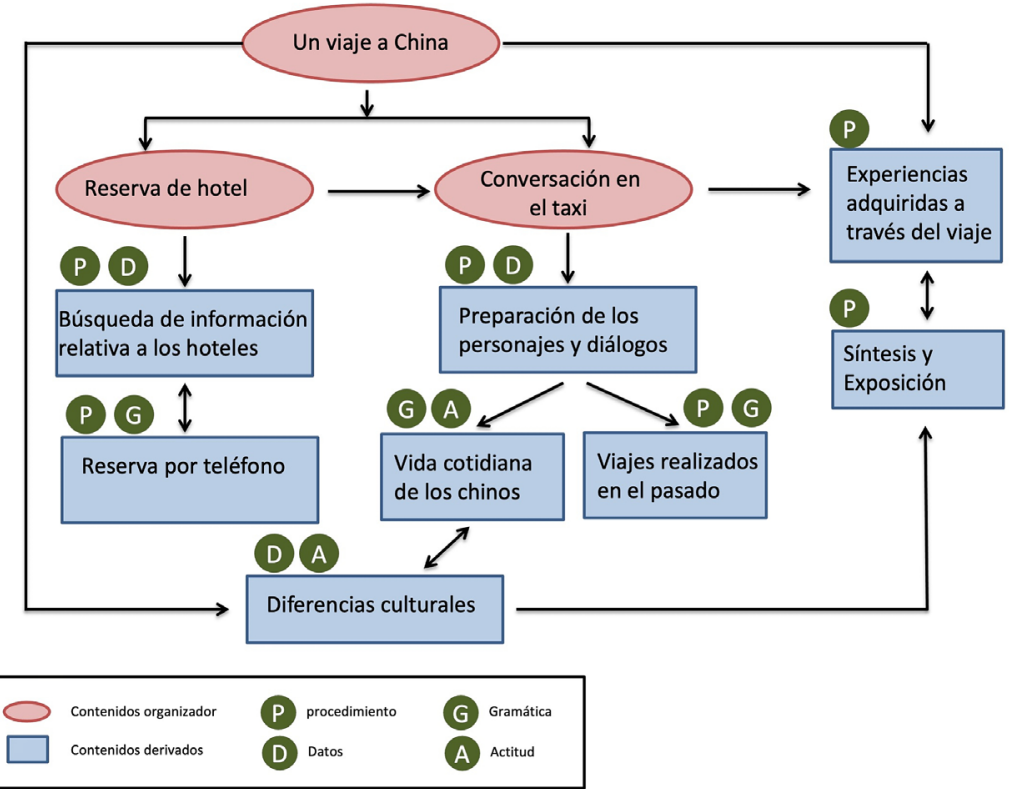

Figura 1. Mapa de contenidos

Los contenidos organizadores o conceptuales se estructuran en 2 partes:

1. Realización de una reserva de hotel tanto como viajero y como recepcionista. Deben saber formular preguntas y contestaciones de los precios de los diferentes tipos de habitaciones, la duración de la reserva (fechas) y la ubicación del hotel. En esta parte de contenidos se trabajarán las aptitudes comunicativas y lingüísticas de este ámbito. Saber formular cuestiones con el adverbio 多 + adjetivo; saber las diferentes formas para expresar un número aproximado: 大概, 左右, y dos números seguidos; uso de preposiciones 从...到... y 离 para expresar la distancia temporal y espacial.

2. Conversación en el taxi. Por un lado, realizamos conversaciones de viajes realizados formulando preguntas con Cuándo, cómo, con quién, etc. Se prestará especial atención en el uso de la estructura是...的

Ciclos de Mejora en el Aula (2020). Experiencias de Innovación Docente de la US Esta obra se distribuye con la licencia Creative Commons 
para la enfatización de elementos en oraciones en el pasado. Por otro lado, hablamos sobre la vida cotidiana de un trabajador chino, en este caso, el taxista. Se repasarán las distintas formas de expresar horarios y actividades que se realizan una persona con frecuencia. El único punto nuevo será la introducción del uso del adverbio incluso/ ni si quiera 连.... 也/都...

También se introducen los contenidos procedimentales para que los estudiantes averigüen por ellos mismos los procedimientos para buscar y reservar un hotel en China por internet, teniendo en cuenta las características y exigencias del usuario; los procedimientos para realiza una reserva de hotel por teléfono; y los procedimientos de búsqueda de información y síntesis sobre la diferencia cultural en el ámbito de negocio.

En cuanto a los contenidos actitudinales, se trabaja en la comprensión del estilo de vida de los trabajadores chinos, en actitudes de respeto enfocando a las diferencias socioculturales manifestados en un viaje de negocio, y en capacidades para enfrentarse a estas situaciones interculturales.

\section{Modelo metodológico posible}

Para la puesta en práctica de este ciclo de mejora, se ha elaborado un modelo metodológico de enseñanza y aprendizaje basado en la resolución de problemas. Un problema enigmático puede ser una herramienta de enseñanza muy útil que estimula la curiosidad, activa el razonamiento y obliga a los alumnos a cuestionar su propia respuesta y pensar más allá (Finkel, 2008). De esta manera, el aprendizaje es mucho más duradero. Por otro lado, el modelo metodológico más empleado en la enseñanza de Ciclos de Mejora en el Aula (2020). Experiencias de Innovación Docente de la US
Esta obra se distribuye con la licencia Creative Commons 
lenguas extranjeras es el modelo comunicativo basado en contextos reales o situaciones simuladas. Por tanto, nuestro diseño busca un modelo didáctico mucho más dinámico y participativo que se centra en los alumnos y sus necesidades.

El modelo metodológico que se ha empleado en el ciclo de mejora se estructura en 4 fases: la primera consiste en una pequeña introducción donde se plantea el problema o la situación inicial (P). La segunda fase consiste en trabajar en grupo de 3 o 4 alumnos para intentar resolver este problema (RP). A continuación, se explicará las dudas que les hayan podido surgir en la actividad anterior. Esta explicación teórica (T) sobre las cuestiones gramaticales de la lengua china les ayudará contractar y mejorar las respuestas del problema inicial. La última fase es la realización de una sintesis (S) respondiendo a la pregunta inicial. Y el proceso del ciclo de mejora termina con una puesta en común que será la preparación de una comunicación oral de todas situaciones planteadas.

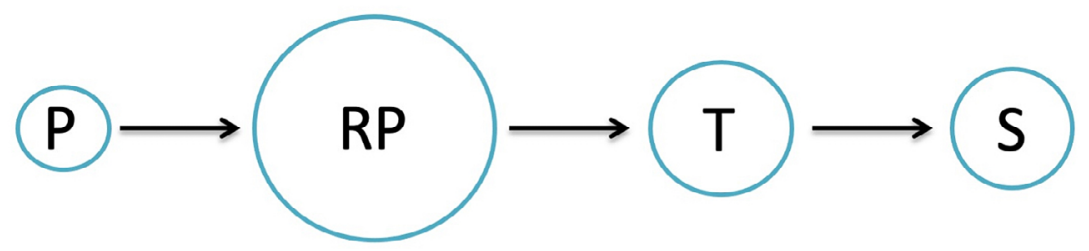

Figura 2. Modelo metodológico posible

\section{Secuencia de actividades programadas}

\section{SESIÓN 1: día 26 de octubre}

Situación inicial: Después de graduarte en el Grado en Estudios de Asia Oriental, has encontrado un trabajo en una pequeña empresa española de aceite de oliva. Una empresa china ha puesto en contacto con vosotros porque tiene intención de importar vuestros productos para su posterior venta en China. Necesitáis ir personalmente alli para concretar los últimos detalles y finalizar el contrato. 
Tú y tu jefe irán los próximos días a Shanghai con un presupuesto limitado para este viaje. Tus tareas consisten en ser traductor, intérprete, consultor cultural, etc. Además, administrar adecuadamente este presupuesto para la reserva del hotel, la comida, transporte y posibles gastos extras.

Presupuesto total: 1000 euros para la estancia de 3 días. Dirección de la empresa: 233 Taicang Rd, Huangpu, China. 新茂大厦.

Llegada: Shanghai Pudong International Airport. 上海浦 东国际机场

Realiza un informe que justifique el gasto total del hotel con las especificaciones del hotel elegido (fecha, precio, distancia al aeropuerto y a la empresa, ambiente, limpieza, servicios adicionales contratados, opiniones, etc.)

Tabla 1. Secuencia de actividades desarrolladas en la primera sesión

\begin{tabular}{|l|l|}
\hline SESIÓN 1: Búsqueda del hotel adecuado y sus características \\
\hline 15 min. & Cuestionario inicial \\
\hline 10 min. & $\begin{array}{l}\text { Planteamiento del problema inicial y explicaciones de los } \\
\text { objetivos a conseguir. }\end{array}$ \\
\hline 45 min. & Trabajo en grupo para encontrar el hotel apropiado. \\
\hline 40 min. & $\begin{array}{l}\text { Explicación de la gramática y expresiones lingüísticas. } \\
\text { Realización del informe que justifique la elección y los } \\
\text { gastos del hotel. } \\
\text { Entrega del informe. }\end{array}$ \\
\hline
\end{tabular}

SESIÓN 2: día 27 de octubre

Una vez decidido en qué hotel os vais a alojar, procederemos a la reserva de dicho hotel. Sin embargo, debido a un problema informático del hotel, solo es posible hacer la reserva por vía telefónica. Por lo cual, la primera mitad

Ciclos de Mejora en el Aula (2020). Experiencias de Innovación Docente de la US Esta obra se distribuye con la licencia Creative Commons 
de esta clase se dedica a la creación de la conversación para la reserva del hotel, teniendo en cuenta los datos obtenidos en la sesión anterior.

Al finalizar esta tarea, tu jefe te llama y te enseña unas corbatas que había comprado como regalo para los clientes chinos. ¿Qué opinas sobre este regalo? ¿Qué regalo elegirías tú? Dale una breve explicaciones de los aspectos socioculturales enfocados al ámbito de negocios que debe tener en cuenta.

Tabla 2. Secuencia de actividades desarrolladas en la segunda sesión

\begin{tabular}{|l|l|}
\hline SESIÓN 2: Reserva del hotel y cuestiones interculturales \\
\hline 15 min. & $\begin{array}{l}\text { Repaso de los contenidos de la sesión anterior. } \\
\text { Planteamiento del segundo problema: realizar la reserva por } \\
\text { teléfono. }\end{array}$ \\
\hline 30 min. & $\begin{array}{l}\text { Preparación y realización del dialogo de la reserva del hotel } \\
\text { por teléfono. }\end{array}$ \\
\hline 15 min. & $\begin{array}{l}\text { Planteamiento del tercer problema: diferencias socioculturales. } \\
\text { Hipótesis de los alumnos. }\end{array}$ \\
\hline 30 min. & $\begin{array}{l}\text { Actividad de contraste: Búsqueda de información sobre las } \\
\text { diferencias culturales y etiquetas para hacer negocios en } \\
\text { China. }\end{array}$ \\
\hline 15 min. & $\begin{array}{l}\text { Puesta en común de la información encontrada. } \\
\text { Contrastar con las hipótesis iniciales. }\end{array}$ \\
\hline
\end{tabular}

SESIÓN 3: día 3 de noviembre

Al llegar al aeropuerto de Shanghai, cogéis un taxi para ir al hotel reservado. El taxista es una persona muy extrovertida y curiosa, tiene muchas aficiones, además de ser muy trabajador como todos los chinos. Trabaja muchas horas al día, ya que es hijo único y tiene que mantener su familia. Y tú eres una persona muy aventurera, has viajado por muchos países, te encantan las distintas culturas.

Ciclos de Mejora en el Aula (2020). Experiencias de Innovación Docente de la US Esta obra se distribuye con la licencia Creative Commons 
Objetivo taxista: averiguar en qué países ha estado el viajero, cuándo y cómo fue estos viajes. Dónde y cuándo estudió el idioma chino.

Objetivo viajero: averiguar el horario de trabajo del taxista, sus hobbies y qué hace en su tiempo libre.

Cada alumno tiene que diseñar su propio personaje para luego establecer la conversación con el otro y conseguir su objetivo.

Tabla 3. Secuencia de actividades desarrolladas en la tercera sesión

\begin{tabular}{|l|l|}
\hline SESIÓN 3: Conversaciones en el taxi \\
\hline 5 min. & $\begin{array}{l}\text { Planteamiento de la nueva situación: En el taxi de camino al } \\
\text { hotel. }\end{array}$ \\
\hline $20 \mathrm{~min}$. & Preparación de los personajes. \\
\hline $15 \mathrm{~min}$. & Realización del dialogo entre el viajero y el taxista. \\
\hline $30 \mathrm{~min}$. & $\begin{array}{l}\text { Actividad de contraste. Explicación de gramática y expresiones } \\
\text { lingüisticas. }\end{array}$ \\
\hline $20 \mathrm{~min}$. & Resumen por escrito del objetivo según cada caso. \\
\hline
\end{tabular}

SESIÓN 4: día 9 de noviembre

Ya has vuelto a España y estas ansioso de contar tu viaje a tu mejor amigo.

Esta actividad se realiza previamente en casa como tarea y se expone por grupo en esta clase. Consiste en contar a los compañeros los sucesos de este viaje, problemas producidos en la reserva o en la estancia del hotel, anécdotas del viaje, conversación con el taxista, curiosidades, etc.

Ciclos de Mejora en el Aula (2020). Experiencias de Innovación Docente de la US Esta obra se distribuye con la licencia Creative Commons 
Tabla 4. Secuencia de actividades desarrolladas en la cuarta sesión

\begin{tabular}{|l|l|}
\hline SESIÓN 4: Exposición y sintesis de la experiencia del viaje \\
\hline 30 min. & $\begin{array}{l}\text { Exposición de un exalumno del grado, sobre su experiencia del } \\
\text { viaje de negocio en China. }\end{array}$ \\
\hline 70 min. & $\begin{array}{l}\text { Exposición por grupo de las experiencias adquiridas a través } \\
\text { de este viaje. } \\
\text { 5-10 minutos por grupo. }\end{array}$ \\
\hline 15 min. & Cuestionario final. \\
\hline
\end{tabular}

\section{Cuestionario inicial - final}

Los cuestionarios iniciales son una herramienta muy útil para conocer los modelos mentales de los estudiantes, nos permiten determinar los conocimientos lingüísticos previos y los obstáculos de aprendizaje. En el cuestionario inicial elaboré cinco preguntas relacionados con los contenidos gramaticales de esta unidad didáctica, cada uno de ellos se enfoca a una cuestión gramatical clave para el aprendizaje de los contenidos de nuestro ciclo de mejora.

Pregunta 1: Describe la ubicación del hotel según el diagrama.

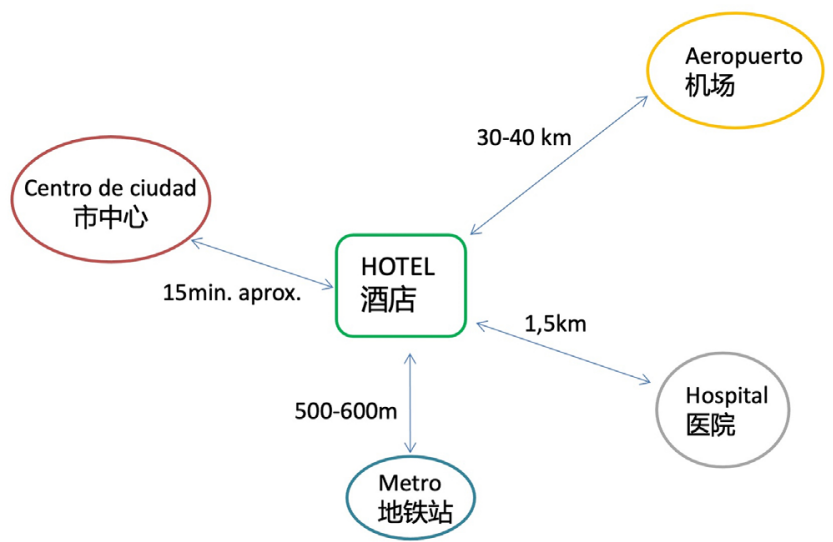

Figura 3. Diagrama de ubicación

Ciclos de Mejora en el Aula (2020). Experiencias de Innovación Docente de la US Esta obra se distribuye con la licencia Creative Commons 
Pregunta 2: Haga una pregunta sobre el precio de las habitaciones y contesta la pregunta. (escribe los números en chino)

Tabla 5. Tipos de habitación

\begin{tabular}{|c|c|}
\hline Estándar Deluxe 豪华标间 & $188 ¥$ \\
\hline Single Deluxe 豪华单间 & $228 ¥$ \\
\hline Habitacion indiviual单人间 & $168 ¥$ \\
\hline Habitacion estandar标准间 & $158 ¥$ \\
\hline
\end{tabular}

Pregunta 3: Según la siguiente tabla de horarios, escribe 3 oraciones especificando la duración de las actividades que realiza Xiaowang habitualmente.

Tabla 6. Actividades de Xiaowang

\begin{tabular}{|l|l|l|l|l|l|}
\hline & 星期一 & 星期二 & 星期三 & 星期四 & 星期五 \\
\hline 17:00-18:00 & 英语 & 英语 & 英语 & 英语 & \multirow{2}{*}{ 骑马 } \\
\cline { 1 - 4 } 18:30-19:30 & 游泳 & 美术(画画) & 游泳 & 美术(画画) & \\
\hline
\end{tabular}

Pregunta 4: Según la información presentada en el billete, formula las siguientes preguntas en chino y contéstalas.

(1) ¿Cuándo fue Zhang Xiaowei a Baoji?

(2) ¿Cómo fue Zhang Xiaowei a Baoji?

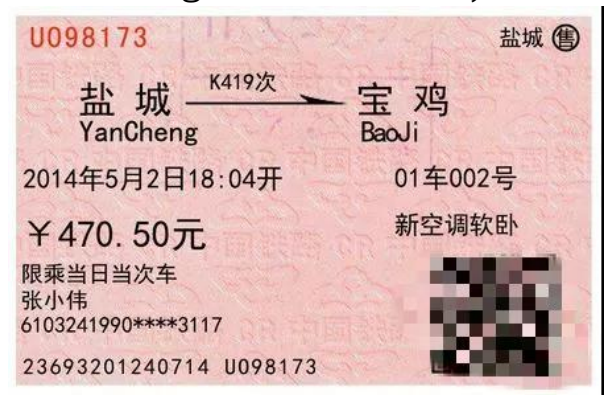

Figura 4. Billete de tren

Ciclos de Mejora en el Aula (2020). Experiencias de Innovación Docente de la US Esta obra se distribuye con la licencia Creative Commons 
Pregunta 5: Traduce las siguientes oraciones.

(1) Estoy tan ocupado que ni siquiera tengo tiempo para descansar los fines de semana.

(2) Algunos caracteres chinos son muy difíciles, hasta los chinos también se equivocan.

\section{Aplicación del CIMA}

\section{Relato resumido de las sesiones}

Siguiendo el diario de clase, en este apartado vamos a explicar de forma resumida el desarrollo real de las actividades planteadas para este ciclo de mejora, incluyendo el clima de trabajo, la practica docente, y los avances y dificultades encontrados.

\section{Sesión 1}

Sólo asistieron 3 alumnos de forma presencial, aunque en este subgrupo hay 10 alumnos. Les entregué el cuestionario, dos de ellos les pareció muy difícil y no pudieron contestar todas las preguntas. Los alumnos que asistían a la clase de forma virtual tenían a su disposición el cuestionario en la plataforma de enseñanza virtual. Algunos alumnos no pudieron terminar el cuestionario a tiempo porque no estaban conectado al principio o tenían problema con la conexión de internet, por tanto, varios de ellos lo entregaron después de la clase.

A continuación, les planteé la primera pregunta o situación: Después de graduarse en el Grado en Estudios de Asia Oriental, trabaja en una pequeña empresa española que vende sus productos en China. Tiene que hacer un viaja los próximos días a Shanghai con un presupuesto limitado. La primera tarea consiste en buscar un hotel

Ciclos de Mejora en el Aula (2020). Experiencias de Innovación Docente de la US Esta obra se distribuye con la licencia Creative Commons 
apropiado en Shanghai teniendo en cuenta la ubicación, el precio, comodidades que ofrece, opiniones etc. En seguida pusieron a trabajar en pequeños grupos. Pero no tenía forma de averiguar si los alumnos que trabajaban en casa estaban realizando correctamente la actividad o no. A la hora de realizar el informe, necesitan justificar la elección del hotel especificando el precio y la ubicación. Por lo cual, necesitan utilizar palabras para expresar números aproximados y distancias. Muchos de ellos tenían ya cierto conocimiento sobre estos aspectos, sólo tuve que explicar brevemente estas cuestiones de la gramática. Al fina de la sesión les pedí que me entregaran el informe por el email.

\section{Sesión 2}

Basándonos en el informe y la búsqueda de información de la sesión anterior, les planteé la segunda situación: Debido a un problema informático del hotel, solo es posible hacer la reserva por vía telefónica. La primera mitad de esta clase se dedicó a la creación de la conversación para la reserva del hotel. Los alumnos presenciales no tuvieron problemas para realizar esta actividad, pero no hubo mucha participación de los alumnos online. Mientras que realizaban el dialogo, paseaba por el aula para escuchar y corregir los fallos que observaba en las conversaciones de los alumnos presenciales. No pude escuchar las conversaciones de los alumnos online, por lo cual, esta actividad hubiera funcionado mejor si la participación fuera de forma totalmente presencial.

En la segunda parte de la sesión, les presenté otra situación: Tu jefe ha elegido unas corbatas como regalo para los clientes chinos. ¿Qué opinas sobre este regalo? ¿Qué regalo elegirías tú? Los alumnos tenían cierto conocimiento sobre el significado de los colores y algunos objetos prohibidos como regalo. A continuación, nos pusimos a leer un artículo sobre el protocolo en la negociación en China, de allí pudieron sacar más información sobre los 
protocolos en el ámbito de negocio y los aspectos culturales que debe tener en cuenta a la hora de tratarse con los chinos. Esta actividad de contraste les ayudó a profundizar sus conocimientos sobre la base cultural e ideológico que dan lugar a estos fenómenos interculturales y sociológicos

\section{Sesión 3}

En esta sesión no vino ningún alumno a clase, por lo cual fue totalmente virtual. Al comienzo de la clase les planteé la nueva situación: Una vez llegado a China, coge un taxi para ir al hotel reservado, ahora surge la conversación entre el viajero y el taxista. Cada alumno tiene que diseñar su personaje y conseguir su objetivo. Cuando se pusieron a trabajar me preguntó un alumno por el chat cómo se decía la palabra taxista, otro alumno contestó la pregunta por el chat. Aunque no participan mucho oralmente, sí que ayudan entre ellos y participan por el chat. Luego hice una explicación sobre la diferencia entre el nombre de la profesión y el nombre que prefieren que les llamemos en la vida real. Este dato les pareció muy interesante y útil ya que sólo en las situaciones reales podrían aprender este tipo de conocimiento.

Para averiguar los viajes que hizo el pasajero, necesitan emplear la estructura gramatical 是......的. Ellos formularon las oraciones en el pasado correctamente, pero sin usar esta estructura gramatical. Aproveché esta oportunidad para explicar la diferencia de estas oraciones con y sin la estructura gramatical 是......的 y luego expliqué brevemente su uso y puse más ejemplos de este tipo de oraciones.

Cuando diseñaban la ajetreada vida del taxista, pusieron frases como "estoy muy ocupado, no tengo tiempo para descansar." Nadie introdujo la conjunción incluso/ ni siquiera 连..... 也/都 para dar énfasis a la oración. Entonces sugerí que podrían usar esta conjunción en la 
conversación, pocos alumnos supieron formular correctamente la oración. A continuación, les di una breve explicación sobre el uso de esta conjunción y después hicimos más ejercicios para practicar esta cuestión gramatical.

Al final de la sesión les pedí que hicieran tres diálogos basándose en las tres situaciones planteadas anteriormente para hacer una exposición oral en la siguiente sesión.

El ambiente de trabajo en esta sesión fue muy bueno, se interesaban por hacer bien las conversaciones, hubo muchas preguntas por el chat. Sin embargo, el no poder escuchar las conversaciones que ellos estaban elaborando, no pude detectar problemas de pronunciación ni de fallos léxicos ni gramaticales.

\section{Sesión 4}

Esta sesión también fue totalmente virtual. Comenzamos con la presentación de un exalumno del Grado de Estudios de Asia Oriental sobre la cultura, el protocolo de negocio en China y algunas expresiones idiomáticas relacionadas. Los alumnos mostraron mucho interés ya que se trataba de una experiencia propia de alguien que ellos se sentían identificados. Después de la presentación siguieron preguntándole sobre las salidas profesionales que pueden haber en China, los másteres relacionados con el grado de estudios asiáticos para poder seguir formándose, etc. Esta actividad duró más tiempo del previsto, pero estoy muy contenta porque mostraron mucho interés y les pareció muy provechosa y práctica la charla.

La segunda parte de la sesión la dediquemos a las exposiciones de los diálogos desarrollados durante las sesiones anteriores, después de cada exposición comentamos algunos errores que observamos en los diálogos, así todos los estudiantes pueden opinar, expresar y aprender de 
estos fallos para no cometerlos en futuras ocasiones. Me hubiera gustado que tuviera más participación, ya que a través del Blackboard no participan mucho.

Finalmente, no hubo tiempo suficiente para terminar las exposiciones, y 3 grupos tuvieron que exponerlos en la siguiente clase. Tampoco hubo tiempo para realizar el cuestionario final, lo hicieron después de la clase y me lo enviaron por el correo electrónico.

\section{Evaluación del aprendizaje de los estudiantes}

Al finalizar una unidad temática, es necesario valorar de nuevo los modelos mentales de los estudiantes y compararlos con los iniciales para conocer el grado de evolución del conjunto de la clase (Rivero y Porlán, 2017). Partimos de los cuestionarios iniciales y finales de los alumnos, hemos elaborado las escaleras de aprendizaje para conocer los avances de los mismos. Como se puede observar, ha habido una mejora en su grado de conocimiento sobre estas cuestiones lingüísticas.

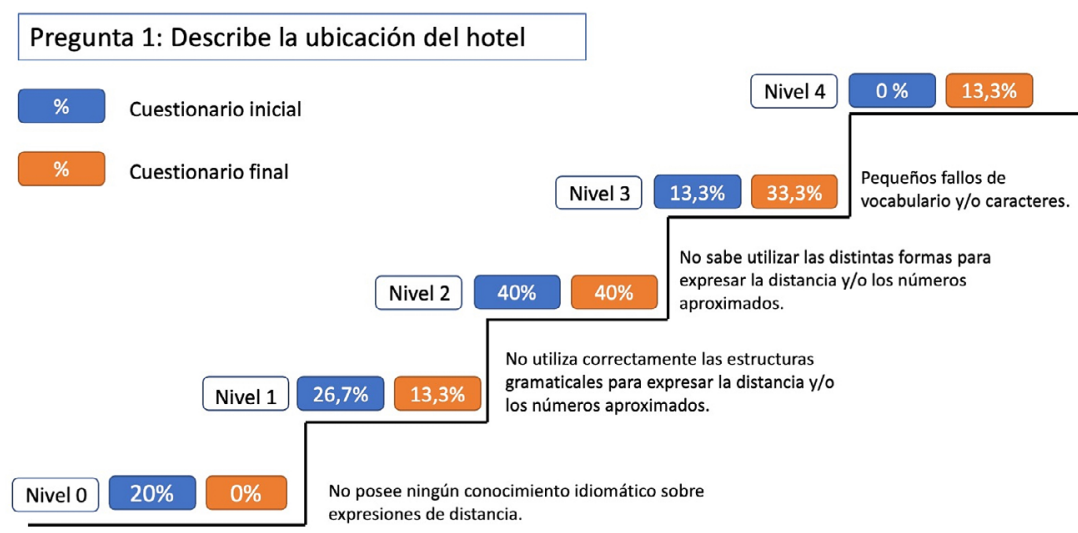

Figura 5. Escalera de aprendizaje de la pregunta 1

Ciclos de Mejora en el Aula (2020). Experiencias de Innovación Docente de la US Esta obra se distribuye con la licencia Creative Commons 
Pregunta 2: Precios de las habitaciones del hotel

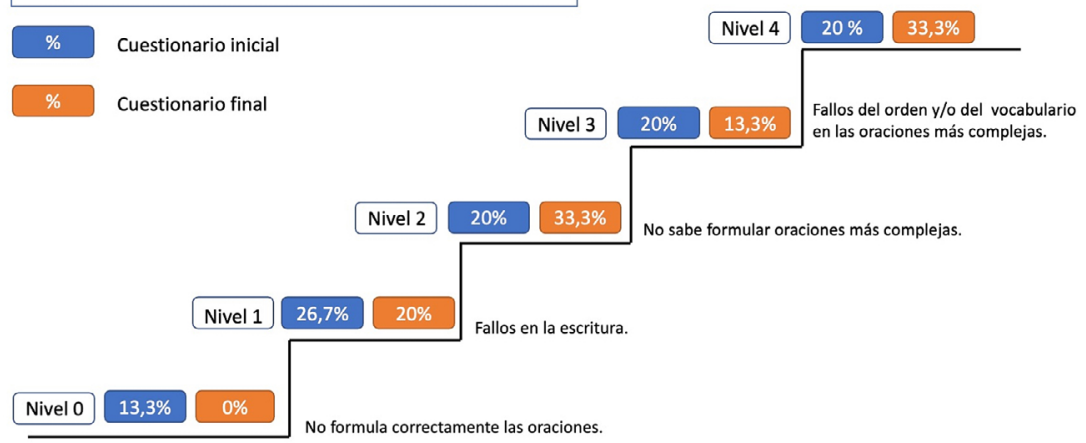

Figura 6. Escalera de aprendizaje de la pregunta 2

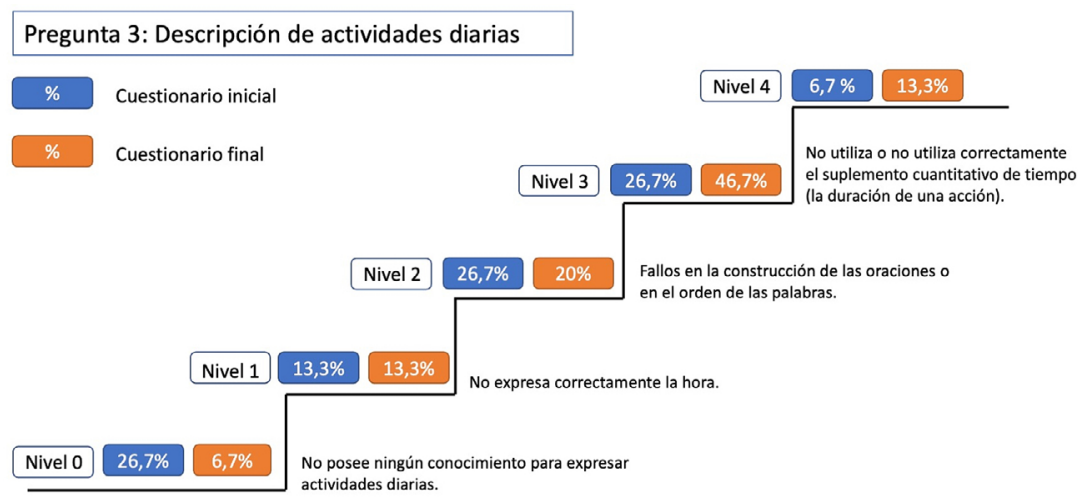

Figura 7. Escalera de aprendizaje de la pregunta 3

Pregunta 4: Formula preguntas en base al billete de tren y contéstalas.

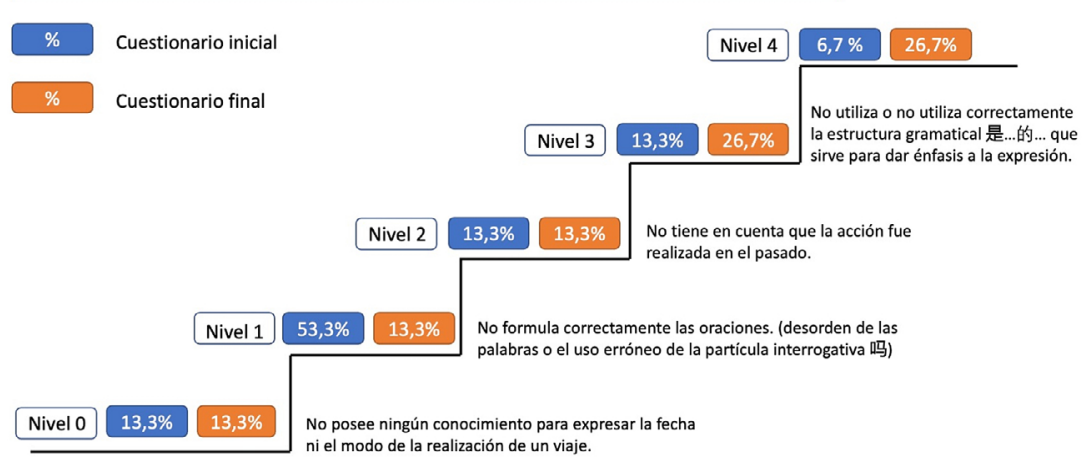

Figura 8. Escalera de aprendizaje de la pregunta 4

Ciclos de Mejora en el Aula (2020). Experiencias de Innovación Docente de la US (c) Esta obra se distribuye con la licencia Creative Commons Reconocimiento-NoComercial-SinObraDerivada $\quad 4.0$ Internacional (CC BY-NC-ND 4.0.) 
Pregunta 5: Traducir las oraciones.

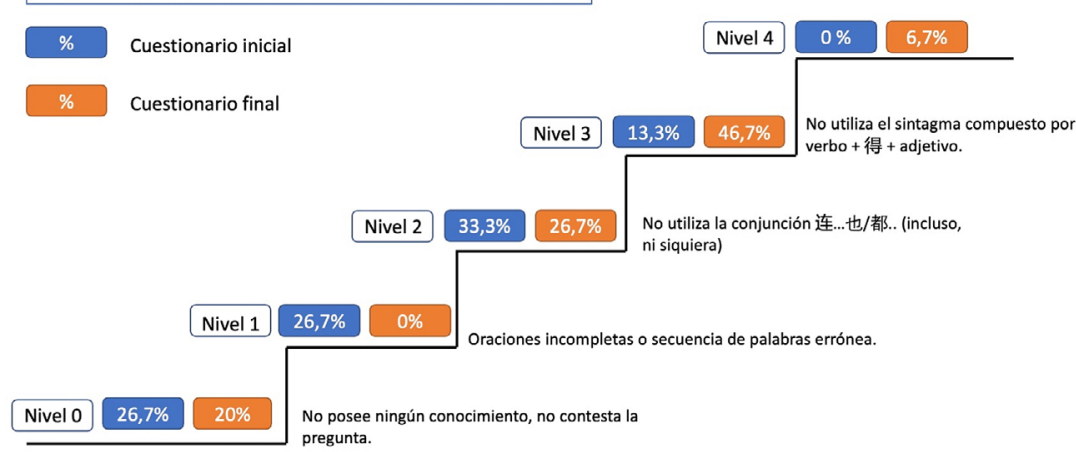

Figura 9. Escalera de aprendizaje de la pregunta 5

Al tratarse de contenidos lingüísticos, si los alumnos no poseen conocimientos lingüísticos previos ni dominan estructuras gramaticales simples, es muy difícil llegar a las más complejas. Por tanto, la evolución del aprendizaje es progresiva.

A continuación, mostramos los avances individuales de cada alumno. Podemos observar que la gran mayoría aumentó el nivel de sus respuestas y algunos mantuvieron el nivel.

Tabla 7 y 8. Evolución de cada alumno

\begin{tabular}{|c|c|c|c|c|c|c|}
\hline Sujeto & \multicolumn{3}{|c|}{ PREGUNTA 1 } & \multicolumn{3}{c|}{ PREGUNTA 2 } \\
\hline 1 & Nivel 3 & Nivel 3 & $\rightarrow$ & Nivel 4 & Nivel 4 & $\leftrightarrow$ \\
\hline 2 & Nivel 0 & Nivel 1 & 4 & Nivel 0 & Nivel 2 & +4 \\
\hline 3 & Nivel 1 & Nivel 1 & $\leftrightarrow$ & Nivel 1 & Nivel 1 & $\leftrightarrow$ \\
\hline 4 & Nivel 2 & Nivel 3 & 4 & Nivel 2 & Nivel 2 & $\leftrightarrow$ \\
\hline 5 & Nivel 0 & Nivel 2 & $4+4$ & Nivel 1 & Nivel 1 & $\leftrightarrow$ \\
\hline 6 & Nivel 2 & Nivel 2 & $\leftrightarrow$ & Nivel 1 & Nivel 2 & 4 \\
\hline
\end{tabular}

Ciclos de Mejora en el Aula (2020). Experiencias de Innovación Docente de la US Esta obra se distribuye con la licencia Creative Commons Reconocimiento-NoComercial-SinObraDerivada Internacional (CC BY-NC-ND 4.0.) 


\begin{tabular}{|c|c|c|c|c|c|c|}
\hline 7 & Nivel 2 & Nivel 3 & 4 & Nivel 2 & Nivel 3 & 4 \\
\hline 8 & Nivel 1 & Nivel 2 & 4 & Nivel 4 & Nivel 4 & $\leftrightarrow$ \\
\hline 9 & Nivel 2 & Nivel 2 & $\leftrightarrow$ & Nivel 3 & Nivel 3 & $\leftrightarrow$ \\
\hline 10 & Nivel 3 & Nivel 4 & 4 & Nivel 3 & Nivel 4 & 4 \\
\hline 11 & Nivel 2 & Nivel 4 & 44 & Nivel 4 & Nivel 4 & $\leftrightarrow$ \\
\hline 12 & Nivel 2 & Nivel 2 & $\leftrightarrow$ & Nivel 2 & Nivel 2 & $\leftrightarrow$ \\
\hline 13 & Nivel 1 & Nivel 2 & 4 & Nivel 1 & Nivel 2 & 4 \\
\hline 14 & Nivel 1 & Nivel 3 & +4 & Nivel 3 & Nivel 4 & 4 \\
\hline 15 & Nivel 0 & Nivel 3 & 444 & Nivel 0 & Nivel 1 & 4 \\
\hline
\end{tabular}

\begin{tabular}{|c|c|c|c|c|c|c|}
\hline Sujeto & \multicolumn{3}{|c|}{ PREGUNTA 3} & \multicolumn{3}{|c|}{ PREGUNTA 4} \\
\hline 1 & Nivel 2 & Nivel 3 & 4 & Nivel 3 & Nivel 3 & $\leftrightarrow$ \\
\hline 2 & Nivel 2 & Nivel 3 & 4 & Nivel 1 & Nivel 2 & 4 \\
\hline 3 & Nivel 0 & Nivel 3 & $4+4$ & Nivel 0 & Nivel 0 & $\leftrightarrow$ \\
\hline 4 & Nivel 3 & Nivel 3 & $\leftrightarrow$ & Nivel 1 & Nivel 1 & $\leftrightarrow$ \\
\hline 5 & Nivel 0 & Nivel 0 & $\leftrightarrow$ & Nivel 0 & Nivel 0 & $\leftrightarrow$ \\
\hline 6 & Nivel 3 & Nivel 3 & $\leftrightarrow$ & Nivel 2 & Nivel 4 & 44 \\
\hline 7 & Nivel 3 & Nivel 3 & $\leftrightarrow$ & Nivel 1 & Nivel 3 & +4 \\
\hline 8 & Nivel 3 & Nivel 3 & $\leftrightarrow$ & Nivel 3 & Nivel 3 & $\leftrightarrow$ \\
\hline 9 & Nivel 1 & Nivel 1 & $\leftrightarrow$ & Nivel 1 & Nivel 1 & $\leftrightarrow$ \\
\hline 10 & Nivel 2 & Nivel 4 & $4+$ & Nivel 1 & Nivel 4 & $+4+$ \\
\hline 11 & Nivel 4 & Nivel 4 & $\leftrightarrow$ & Nivel 1 & Nivel 4 & $4+4$ \\
\hline 12 & Nivel 0 & Nivel 2 & 44 & Nivel 2 & Nivel 3 & 4 \\
\hline
\end{tabular}

Ciclos de Mejora en el Aula (2020). Experiencias de Innovación Docente de la US Esta obra se distribuye con la licencia Creative Commons 


\begin{tabular}{|c|c|c|c|c|c|c|}
\hline 13 & Nivel 1 & Nivel 1 & $\leftrightarrow$ & Nivel 1 & Nivel 2 & 4 \\
\hline 14 & Nivel 0 & Nivel 2 & +4 & Nivel 4 & Nivel 3 & 4 \\
\hline 15 & Nivel 2 & Nivel 2 & $\leftrightarrow$ & Nivel 1 & Nivel 4 & $4+4$ \\
\hline
\end{tabular}

\section{Evaluación del CIMA}

La evaluación de este ciclo de mejora parte de los resultados de aprendizaje de los estudiantes y de un ejercicio de reflexión sobre la puesta en práctica del CIMA. En general podemos afirmar que el resultado de este ciclo de mejora ha sido satisfactorio, y como muestra de ello, los estudiantes han mejorado en general sus conocimientos lingüísticos sobre los contenidos de esta unidad didáctica. Sin embargo, la participación ha reducido considerablemente debido al modo virtual de la docencia, convendría mejorar las estrategias para fomentar la práctica oral en clase.

En el cuestionario final los estudiantes expresaron sus opiniones sobre este ciclo de mejora. A todos ellos les ha parecido mucho mejor este método didáctico por diferentes razones. Pero los alumnos con poco nivel de base, les han parecido muy difíciles las prácticas, ya que no tienen la base de conocimiento suficiente para realizarlas.

En cuanto al aprendizaje de los alumnos, considero muy importante centrar nuestra enseñanza en los alumnos, conocer sus modelos mentales, de esta forma podemos detectar los niveles y los obstáculos de los estudiantes, para posteriormente adaptar los contenidos y la metodología de enseñanza. Mantendré el uso del cuestionario inicial en los futuros ciclos de mejoras, ya que es una herramienta muy eficaz que aportan datos muy útiles para mejorar la enseñanza y el aprendizaje.

Ciclos de Mejora en el Aula (2020). Experiencias de Innovación Docente de la US Esta obra se distribuye con la licencia Creative Commons 
En cuanto a los contenidos de enseñanza, representar el mapa de contenidos y problemas esquemáticamente me han ayudado organizar los contenidos para que tengan continuidad y enfocarlos para que tengan utilidad práctica en situaciones reales y cotidianas, así como la relación entre los diferentes tipos de contenido.

En cuanto a la metodología considero muy importantes las preguntas, deben ser interesantes, atractivas y cercanas a la vida cotidiana, de esta manera, los estudiantes se concentran, se esfuerzan y aprenden más. Normalmente en mis clases, primero explico la gramática y luego hacemos ejercicios escritos y orales para practicar esta gramática. En el CIMA, el orden de las actividades ha sido al revés, primero se plantea el caso práctico, para que ellos reflexionen sobre las estructuras gramaticales necesarias en esta situación, y luego completo la actividad con una breve explicación de la gramática. De esta forma, los estudiantes se acuerdan mejor de las explicaciones porque ya habian reflexionado sobre ellas, además, las situaciones planteadas les ofrecen un acercamiento al uso real de la lengua china, que es considerado el objetivo del aprendizaje de un idioma.

En cuanto a la evaluación, los cuestionarios iniciales y finales me han permitido conocer los niveles, dificultades y avances que ha tenido cada alumno, y las escaleras de aprendizaje para mi han sido una manera novedosa y revolucionaria de evaluación. Gracias a estas herramientas, ahora puedo realizar un seguimiento de cada alumno y saber si mi método didáctico funciona o no.

Ciclos de Mejora en el Aula (2020). Experiencias de Innovación Docente de la US Esta obra se distribuye con la licencia Creative Commons 
Palabras Clave: Chino, Grado en Estudios de Asia Oriental, docencia universitaria, experimentación docente universitaria.

Keywords: Chinese, Degree in East Asian Studies, university teaching, university teaching experimentation.

\section{Referencias bibliográficas}

Bain, K. (2007). Lo que hacen los mejores profesores universitarios. Valencia: Universitat de València.

Finkel, D. (2008). Dar clase con la boca cerrada. Valencia: Universitat de València.

Porlán, R. (2017). Enseñanza universitaria. Cómo mejorarla. Madrid: Morata.

Rivero, A. y Porlán, R (2017). La evaluación en la enseñanza universitaria. En R. Porlán, (Coord.), Enseñanza universitaria. Cómo mejorarla (pp. 73-91). Madrid: Morata.

Ciclos de Mejora en el Aula (2020). Experiencias de Innovación Docente de la US Esta obra se distribuye con la licencia Creative Commons 